\title{
Calculating appropriate target cancer detection rates and expected interval cancer rates for the UK NHS Breast Screening Programme
}

\author{
Sue Moss, Roger Blanks for the Interval Cancer Working Group
}

\begin{abstract}
Objectives-To enable better monitoring of interim outcome measures in the NHS Breast Screening Programme by providing revised targets for cancer detection rates, and revised expected interval cancer rates.

Design and setting-Expected detection rates of invasive cancers at prevalent screen are calculated, using estimates of the underlying England and Wales incidence rates and age specific prevalence :incidence ratios from the Swedish Two County Study. Expected interval cancer rates are also derived from this study, and are used to calculate expected detection rates at rescreening.

Results-The expected invasive cancer detection rates at first screen for women aged $50-52$ is 3.6 per 1000 . The expected rate at rescreening for women aged 53-64 is 4.0 per 1000 . Expected interval cancer rates for women screened from 1995/6 onwards are 0.45 per $1000,0.65$ per 1000 , and 1.2-1.3 per 1000 for the periods within $0-<12,12-<24$, and $24-<36$ months of screening.

Conclusions-The target cancer detection rates and expected interval cancer rates for the NHS Breast Screening Programme have been revised in the light of more recent data. Monitoring of the extent to which the programme is meeting these revised targets will give a more accurate indication of the potential to meet the Health of the Nation target of a $25 \%$ reduction in breast cancer mortality by the year 2000 .
\end{abstract}

\section{(F Epidemiol Community Health 1998;52:111-115)}

It will be many years after the introduction of a breast screening programme before the outcome in terms of a reduction in mortality from breast cancer can be expected. In the meantime, it is important that interim outcome measures are monitored to determine if the programme is performing satisfactorily. One of the main such outcome measures will be the cancer detection rate, both at first ("prevalent") and subsequent ("incident") screens. The rate of interval cancers arising in the period after a negative screen is also an important interim outcome measure of the effect of screening. It provides information on the sensitivity of the screening being carried out, and on the probable future impact of screening on mortality. Targets were also set for the first time for the incident screening round.

The NHS Breast Screening Programme, established in 1988 on the recommendations of the Forrest Committee, ${ }^{1}$ has always recognised the need for quality assurance of all aspects of the programme. In 1989, guidelines for quality assurance of mammography were published, ${ }^{2}$ which included outcome objectives to be assessed by "acceptable values" for a number of measurements (table 1), including the cancer detection rate at prevalent screens.

In January 1993, updated objectives were published, ${ }^{3}$ which included both "acceptable" and "achievable" standards for a number of criteria. The acceptable value for referral for assessment was reduced from less than $10 \%$ to less than $7 \%$, and a target malignant to benign biopsy ratio of at least 1:3 was changed to a ratio of at least $1: 1$, the latter two targets applied to both prevalent and incident screens. These figures are also shown in table 1 . Neither document specified explicitly how the standards had been arrived at, although they were largely based on experience from research trials of breast screening by mammography, notably the Swedish Two County study, which achieved a $30 \%$ mortality reduction after seven years of follow up. ${ }^{4}$

Expected rates of interval cancers after negative screens were also published in $1993,{ }^{5}$ together with definitions of what should be included as interval cancers. Again these were based on the interval cancer rates observed in the Swedish Two County study, ${ }^{6}$ but applied to the estimated underlying annual incidence rate for the UK. It is anticipated that the NHS Breast Screening Programme, within its current three year screening interval, will need to achieve similar rates to reach the Health of the Nation target of a $25 \%$ reduction in breast cancer mortality by the year 2000 .

A working group set up in 1994 has been re-examining all these targets in the light of greater experience and updated information. In particular, it is now recognised that targets for cancer detection rates and estimates of interval cancer rates should apply to invasive cancers only. This is because the underlying incidence rates used to derive the targets will in general be those for invasive disease. Microinvasive cases should also be excluded from the invasive cancer rates. 
Table 1 Previous NHSBSP targets

\begin{tabular}{lll}
\hline & $1989^{2}$ & $1993^{3}$ \\
\hline Prevalent screen & $<10 \%$ & \\
$\quad$ Referral rate & $>5$ per 1000 & $>7 \%$ \\
$\quad$ Cancer detection rate & $>1.5$ per 1000 & $>1.5$ per 1000 \\
$\quad \begin{array}{l}\text { Detection of cancer < } 10 \mathrm{~mm} \\
\text { Incident screen } \\
\quad \text { Cancer detection rate }\end{array}$ & - & $>3.5$ per 1000 \\
Prevalent incident & $>1: 3$ & $>1: 1$ \\
$\quad$ Malignant:benign ratio & $<6$ per 10000 & $<3$ per 10000 \\
\hline
\end{tabular}

Invasive cancer detection rates at first screen

The expected cancer detection rate at the first ("prevalent") screen is dependent on the underlying, or background, incidence rate and on the prevalence to incidence ratio, taking into account the sensitivity of screening. Both of these factors vary with the age of the women being screened.?

Now that the NHS Breast Screening Programme is well established, women being invited for screening for the first time will be aged between 50 and 52 , because those aged 53 and over should already have received at least one invitation. The average age of new invitees will therefore be approximately 51 years 6 months.

UNDERLYING INCIDENCE RATES

The underlying incidence rate of breast cancer for England and Wales has in the past been taken from the Office of Population Censuses and Surveys (OPCS) published data. However, a recent exercise undertaken by Prior et $a l^{8}$ from which more complete data from cancer registries have been compiled, showed that the OPCS figures may underestimate the true incidence by approximately $10 \%$. The updated data, together with OPCS data, where available, are shown in table 2 for the period 1980 1992.

There has been an increasing trend in incidence over the entire period, but the rate of increase from 1988 onwards is greater because of the impact of the screening programme. Therefore 1987 is the last year that can be used to provide information on incidence rates in the absence of widespread screening. Using data for this year, and assuming a log-linear relation between age (between 50 and 64) and incidence rate, gives an estimated true background incidence rate for women aged 51.5 years of 1.60 per 1000 .
It is possible to extrapolate the trend for 1980-1987 onward to 1995 , giving an estimate of 1.86 per 1000 . Because of the uncertainty surrounding such extrapolation, the midpoint of the two estimates has been used, giving a rate of 1.73 per 1000 . Clearly for years other than 1995, the estimates will differ slightly.

PREVALENCE:INCIDENCE RATIO

Data from the Swedish Two County Study have been used to provide estimates of the prevalence:incidence ratio. Data from the UK Trial of Early Detection of Breast Cancer (TEDBC) are not applicable because screening included both mammography and physical examination. ${ }^{9}$ However, it should be noted that the Two County Study used single view mammography, whereas in the NHS Breast Screening Programme approximately half the programmes have been using two views in the prevalent round, and all are being advised to do so in the future. This is because of evidence, both from the UKCCCR trial of one compared with two view mammography ${ }^{10}$ and from the results of the NHS Breast Screening Programme ${ }^{11}$ that screening by two views in the prevalent round leads to a higher cancer detection rate, as well as a lower recall rate to assessment. The prevalence:incidence ratios from the Two County study may therefore underestimate those we should expect.

The age specific prevalence:incidence ratios for the Swedish Two County Study (Duffy, personal communication, 1995) for invasive cancer only are from table 3 . (The variation in this ratio with age will be caused by differences both in sensitivity and in the mean pre-clinical duration of the disease; it is not possible to separate the two effects)

Again assuming a log-linear relation with age, the estimated prevalence:incidence ratio at age 51.5 is 2.10 .

Multiplying this figure by the estimated background incidence of 1.73 per 1000 derived earlier gives a predicted cancer detection rate of 3.63 per 1000 for women aged 50-52.

To allow past rates, and also those in older women who failed to attend for screening at first invitation, to be compared with the new targets, the methods described above have been used to calculate expected cancer detection rates in other age groups. These are also shown in table 3.

Table 2 Published and revised invasive breast cancer incidence rates per 10000 women

\begin{tabular}{|c|c|c|c|c|c|c|c|c|}
\hline \multirow[b]{2}{*}{ Age group } & \multicolumn{2}{|l|}{$50-54$} & \multicolumn{2}{|l|}{$55-59$} & \multicolumn{2}{|l|}{$60-64$} & \multicolumn{2}{|c|}{ Total 50-64 } \\
\hline & OPCS & Revised & OPCS & Revised & OPCS & Revised & OPCS & Revised \\
\hline 1980 & & 14.53 & & 18.44 & & 19.46 & & 17.45 \\
\hline 1981 & 13.91 & 15.20 & 16.22 & 17.87 & 17.18 & 18.94 & & 17.34 \\
\hline 1982 & 14.59 & 15.71 & 16.65 & 18.19 & 18.52 & 20.38 & & 18.11 \\
\hline 1983 & 13.90 & 15.47 & 16.39 & 18.15 & 17.45 & 19.72 & & 17.84 \\
\hline 1984 & 13.97 & 15.40 & 16.53 & 18.24 & 18.11 & 19.80 & 16.28 & 17.90 \\
\hline 1985 & 14.38 & 16.30 & 16.03 & 18.04 & 19.29 & 21.68 & 16.64 & 18.75 \\
\hline 1986 & 15.44 & 16.99 & 16.77 & 18.23 & 19.79 & 21.45 & 17.38 & 18.93 \\
\hline 1987 & 14.95 & 16.69 & 17.24 & 19.05 & 20.90 & 22.91 & 17.72 & 19.58 \\
\hline 1988 & & 17.55 & & 20.15 & & 23.79 & & 20.50 \\
\hline 1989 & & 18.42 & & 22.42 & & 25.80 & & 22.17 \\
\hline 1990 & & 20.12 & & 24.20 & & 29.71 & & 24.61 \\
\hline 1991 & & 22.44 & & 27.31 & & 30.51 & & 26.70 \\
\hline 1992 & & 23.18 & & 26.54 & & 29.73 & & 26.42 \\
\hline
\end{tabular}


Table 3 Expected invasive cancer detection rates

\begin{tabular}{llll}
\hline Age group & Expected rates & $P / I$ & $\begin{array}{l}\text { Incidence rate per 1000 } \\
\text { for England and Wales }\end{array}$ \\
\hline $50-52$ & 3.63 per 1000 & 2.10 & 1.73 \\
$53-54$ & 4.44 per 1000 & 2.39 & 1.86 \\
$50-54$ & 3.94 per 1000 & 2.21 & 1.81 \\
$55-59$ & 5.86 per 1000 & 2.85 & 2.06 \\
$60-64$ & 8.72 per 1000 & 3.67 & 2.38 \\
\hline
\end{tabular}

\section{Invasive cancer detection rates at rescreening}

Women being invited for rescreening will be between ages 53 and 64 . The average ages of women in the age band 50-54 will therefore be 54.0. Using the method above gives estimated background incidence rates at this age of 1.86 per 1000 (again taking the midpoint between the 1987 rate and the extrapolated rate for 1995). The rates for women aged 55-59 and 60-64 for 1987 can be taken directly from table 2 , the mean of these values and the extrapolated rates being 20.6 and 23.8 respectively.

Weighting these incidence rates by the estimated populations in 1987 from OPCS data $^{12}$ gives an incidence rate for women aged 53-64 of:

$$
\begin{gathered}
(1.86 \times 519300)+(2.06 \times 1360600) \\
+(2.38 \times 1376300) \\
3256200 \\
=2.16 \text { per } 1000
\end{gathered}
$$

If there were no interval cancers, and assuming no overdiagnosis by screening, the expected detection rate at three yearly rescreening would be three times the annual background incidence. Therefore the cancer detection rate can be estimated by subtracting the expected interval cancers from this figure.

This calculation is based on the theory that an "unbiased set" of cancers from which length bias has been removed can be identified as those occurring in the interval between screens plus those detected at rescreening. ${ }^{13}$ The theory relies on a number of assumptions. It assumes that the incidence of preclinical disease in the interval between screens will be equal to the incidence of clinical disease. It also assumes that the sensitivity of screening will be unaffected by age, and will be the same at rescreening as at the prevalent screen. Further work is planned to investigate the possibility of producing more accurate estimates, although other proposed methods have resulted in similar estimates to that obtained here.

Targets for interval cancers in the NHS Breast Screening Progamme have again been recalculated below using updated data from the Swedish Two County study. Over a three year period these rates are $38 \%$ of the underlying incidence rate. The expected interval cancer detection rate can therefore be calculated as:

$$
\begin{aligned}
& 3 \times 2.16 \times 0.38 \\
& =2.46 \text { per } 1000
\end{aligned}
$$

and the expected cancer detection rate as:

$$
(3 \times 2.16)-2.46
$$

$=4.02$ per 1000

For individual age groups, the expected rates are :
53-54 3.46 per 1000

55-59 3.79 per 1000

60-64 4.42 per 1000

The invasive cancer detection rate targets have therefore been set at 3.6 per 1000 for prevalent screens (that is, women invited for the first time aged 50-52) and 4.0 per 1000 for rescreening in women aged 53-64.

The range of rates that include these targets within the $95 \%$ confidence intervals, for different numbers of women screened is shown in table 4 .

It is recognised that the actual detection rate at rescreening will be influenced by a number of factors including the detection rate at the previous screen, and the rescreening interval. These targets may therefore be less robust than those for the prevalent round, and results from individual programmes will require careful interpretation.

\section{Additional targets}

The revised standard screening office returns (KC62) for the NHS Breast Screening Programme allows calculation of rates of invasive cancers $<10 \mathrm{~mm}$ and $<15 \mathrm{~mm}$ in diameter.

A target of at least $50 \%$ of invasive cancers to be $<15 \mathrm{~mm}$ in diameter has been set, based on data from the Swedish Two County Study. ${ }^{14}$ Calculation of rates of small invasive cancers should exclude microinvasive cancers.

It is expected that between 10 and $20 \%$ of screen detected cancers will be in situ or microinvasive. Missing data on size of cancers cause considerable problems in evaluation of these data.

It is therefore also proposed that a target should be set for the number of invasive cancers of unknown size to be $<=5 \%$.

\section{Interval cancer rates}

The 1993 targets were based on an underlying breast cancer incidence rate for women aged 50 to 64 of 1.7 per 1000 per annum, taken from OPCS data. The incidence of interval cancers after a negative screen in the Swedish Two County Study, in the age-group 50-69 had been reported as $13 \%$ of the underlying incidence in the first year, and $29 \%$ in the second year. This study had an average screening interval of 33 months in this age group, and for the third year period an interval cancer rate of $45 \%$ was observed.

Applying these percentages to the underlying UK incidence rate produced expected rates of $0.22,0.49$, and 0.77 per 1000 for the first, second, and third years after screening. On the basis of these figures, targets were set at $0.2-0.3,0.4-0.5$, and $0.7-0.8$ per 1000 for the three years.

\section{REVISED TARGETS}

As discussed above, it has recently become apparent that OPCS data underestimate the actual UK incidence, and that there was an increasing trend in incidence before 1987. The background incidence rate of 2.16 per 1000 calculated above for women aged 53-64 has been 
Table 4 Observed cancer detection rates that include target rates within $95 \%$ confidence intervals

\begin{tabular}{ll}
\hline \begin{tabular}{l} 
Prevalent screen \\
(women aged 50-52), rate $=3.6$ per 1000 \\
\hline screened
\end{tabular} & $\begin{array}{l}\text { Range of rates including } 3.6 \text { within } 95 \% \\
\text { confidence intervals }\end{array}$ \\
\hline 1000 & $1.3-10.1$ \\
2000 & $1.7-7.5$ \\
3000 & $2.0-6.5$ \\
5000 & $2.3-5.7$ \\
10000 & $2.6-5.0$ \\
25000 & $2.9-4.4$ \\
100000 & $3.2-4.0$ \\
Incident screen & women aged 53-64), rate $=4.0$ per 1000 \\
\hline Number & Range of rates including 4.0 within $95 \%$ \\
screened & confidence intervals \\
\hline 1000 & $1.5-10.7$ \\
2000 & $2.0-8.0$ \\
3000 & $2.3-7.0$ \\
5000 & $2.6-6.2$ \\
10000 & $2.9-5.5$ \\
25000 & $3.3-4.9$ \\
100000 & $3.6-4.4$ \\
\hline
\end{tabular}

used here, although it is recognised that some women will be below 53 and some above age 64 at time of diagnosis of an interval cancer.

In addition, more recent data from the Swedish Two County Study have become available, showing the interval cancer rates for women aged 50-69 at screening to be $14 \%$, $27 \%$, and $55 \%$ of the incidence rate in the control group in the first, second, and third year respectively. However, there are reasons why the UK figures might be expected to be higher than these. Firstly, the upper age limit of women screened in the UK is lower than in the Two County study, and it was observed in the latter that interval cancer rates decreased with age. Secondly, women in the Two County study were rescreened at 33 months, compared with the 36 month interval in the UK. To allow for these differences, percentages of $20 \%, 30 \%$, and $60 \%$ have been used, giving expected rates of $0.43,0.65$, and 1.30 per 1000 for the three 12 month periods.

On this basis, it is proposed that regional and national rates should be compared with expected rates $<=0.45$ per 1000 for interval cancers within $0-<12$ months of screening and $<=0.65$ per 1000 for those within $12-<24$ months, with a combined rate of $<=1.2$ per 1000 for the period $0-<24$ months, and an expected rate for the interval $24-<36$ months of $1.2-1.3$ per 1000 .

Interval cancer rates for individual programmes will in general be based on too small numbers to permit meaningful comparison, and it is anticipated that rates will be evaluated on a regional basis rather than being used to measure the success of individual programmes.

The expected rates calculated here are for overall interval cancer rates, regardless of the age of women screened, or whether they follow a prevalent or incident screen. The calculation of more specific rates is not straightforward, being dependent on variation in both sensitivity and background incidence, and further work is required.

These expected rates apply essentially to interval cancer rates occurring in future years
KEY POINTS

- Based on the Swedish Two County study target invasive detection rates for the NHS Breast Screening Programme are 3.6 per 1000 for prevalent (first) screens in women aged 50-52, and 4.0 per 1000 for incident (subsequent) screens in women aged 53-64.

- Expected interval cancer rates are 1.2 per 1000 for the period $0-<24$ months, and $1.2-1.3$ per 1000 for the interval $24-<36$ months after a negative screen.

- Comparison of observed rates with these expected figures will assist in monitoring the progress of the NHSBSP.

after screening taking place from 1995/6 onwards. There is some evidence that past rates may exceed those expected rates, ${ }^{15}{ }^{16}$ but improvements in screening procedures in the past one or two years, together with the growing experience of screening programmes, should improve sensitivity.

As for cancer detection rate targets, these should be applied to invasive cancers only, because in situ cases are excluded from the underlying incidence rate. (Interval cancers of unknown invasive status should be counted as invasive).

\section{Discussion}

Targets for invasive cancer detection rates are based on figures of 3.6 per 1000 for prevalent screens in women aged 50 to 52 , and 4.0 per 1000 for rescreening in women aged 53 to 64 . In setting these targets, the Swedish Two County study has been used as the "gold standard", because it has achieved the most clear cut reduction in mortality. An attempt has been made to estimate the background incidence in the UK in the absence of screening. It is recognised that different models using more detailed data may produce different predicted rates; however the overall targets are not greatly affected.

In comparing detection rates in women who have previously failed to attend for screening with expected rates, it should be noted that these figures assume that such women have the same background incidence as the general population - that there is no selection biaseither in their initial decision not to attend or in their subsequent attendance. Data on incidence in non-attenders in the NHS Breast Screening Programme are not available for most of the country. Results from the UK TEDBC showed that non-attenders had an increased mortality from breast cancer but not an increased incidence. ${ }^{17}$

It is recognised that targets do not take account of individual programme characteristics, in particular the different age distribution of women screened, and possible variation in the background incidence of breast cancer in each programme's catchment area. In addition, many programmes will be carrying out relatively small numbers of prevalent screens in future years. To measure individual pro- 
gramme performance more accurately a further measure known as the "Standardised Detection Ratio" (SDR) will be introduced and will use the calculated targets for different age groups to provide an age standardised measure of programme performance for invasive cancers. ${ }^{7}$ Proposals have also been made for estimating correction factors to take account of variations in background incidence. $^{18}$

Estimates of interval cancer rates have been recalculated here, again based on results from the Two County study. Interval cancer rates are not always easily estimated from other trials in a similar format. However, the Malmo trial, which showed a $19 \%$ non-significant mortality reduction after nine years in women aged 45-70, had an interval cancer rate of 7.5 per 10000 , or $32 \%$ of the control group rate in the $18-24$ months interval between screens $(27 \%$ if in situ cancers are excluded). ${ }^{19}$ The Stockholm trial, which showed an (again non-significant) $24 \%$ mortality reduction, seems to have an interval cancer rate of 18.5 per 10000 , or $52 \%$ of the control group rate, in the two years after the first screen. ${ }^{20}$ Results from elsewhere are less comparable, either because screening has included clinical examination, or because they lack control populations. However, in general interval cancer rates appear higher than in the Two County Study. Nevertheless, it is probable that the current targets will need to be met if the anticipated reduction in breast cancer mortality is to be achieved.

Funding: The Cancer Screening Evaluation Unit at the Institute of Cancer Research is supported by the Research \& Development Division, Department of Health. Meetings of the Working Group were funded by the NHSBSP.

Conflicts of interest: none.

1 Forrest APM. Breast cancer screening: Report to the Health Ministers of England, Wales, Scotland and Northern Ireland. London: H M Stationery Office, 1986.
2 Quality Assurance Guidelines for Mammography (Pritchard Report). Oxford: NHSBSP, 1989

3 Objectives for the NHSBSP. Oxford: January 1993.

4 Tabar L, Gad A, Holmberg LH, et al. Reduction in mortality from breast cancer after mass screening with mammography. Lancet 1985;i:829-32.

5 Patnick J, Muir Gray JA. Guidelines on the collection and use of breast cancer data. Sheffield: NHSBSP Publication no 26, 1993.

6 Tabar L, Fagerberg G, Day NE, Holmberg L. What is the optimum interval between mammographic screening examinations? Br f Cancer 1987;55:547-51.

7 Blanks RG, Day NE, Moss SM. Monitoring the performance of breast screening programmes: use of indirect standardisation in evaluating the invasive cancer detection rate. $\mathcal{F}$ Med Screen 1996;3:79-81.

8 Prior P, Woodman CBJ, Wilson S, Threlfall AG. Reliability of underlying incidence rates for estimating the effect and efficiency of screening for breast cancer. $7 \mathrm{Med}$ Screen 1996;3:119-22

9 UK Trial of Early Detection of Breast Cancer Group. Trial of early detection of breast cancer: description of method. Br f Cancer 1981;44:618-27.

10 Wald NJ, Murphy P, Major P, Parkes C, Townsend J, Frost C. UKCCCR multicentre randomised controlled trial of one and two view mammography in breast cancer one and two view mammography

11 Moss SM, Michel M, Patnick J, Johns L, Blanks R, Chamberlain J. Results from the NHS breast screening programme 1990-1993. F Med Screen 1995;2:186-90.

12 Mortality statistics: cause. England and Wales 1987. London: OPCS Series DH2, no 14. HMSO, 1989.

13 Duffy SW, Tabar L, Fagerberg G, et al. Breast screening, prognostic factors and survival - results from the Swedish two county study. Br f Cancer 1991;64:1133-8.

14 Day NE, Williams DRR, Kaw KT. Breast cancer screening programmes: the development of a monitoring and evaluation system. Br f Cancer 1989;59:954-8.

15 Woodman CBJ, Threlfall A, Boggis CRM, Prior P. Is the three year breast screening interval too long? Occurrence of interval cancers in NHS breast screening programme's north western region. BMF 1995;310:224-6.

16 Day N, McCann J, Camilleri-Ferrante C, et al. Monitoring interval cancers in breast screening programmes: the East interval cancers in breast screening programmes:

17 UK Trial of Early Detection of Breast Cancer Group. First results on mortality reduction in the UK Trial of Early Detection of Breast Cancer. Lancet 1988;ii:411-16.

18 Blanks RG, Moss SM. Monitoring the performance of the breast screening programmes: allowing for geographical variation in breast cancer incidence. 7 Med Screen 1996;3:82-4.

19 Ikeda DM, Andersson I, Wattsgard C, Janzon L, Linell F. Interval cancinomas in the Malmo mammographic screening trial: radiographic appearance and prognostic considerations. Am f Radiol 1992;159:287-94.

20 Frisell J, Eklund G, Hellstrom L, Somell A. Analysis of interval carcinomas in a randomized screening trial in Stockholm. Breast Cancer Res Treat 1987;3:219-25. 